\title{
A Case of Maculopapular Skin Rash without any Systemic Symptoms
}

Mohammad Mainul Hasan Chowdhury ${ }^{1}$, Farzana Shumy ${ }^{2}$, Probal Sutradhar ${ }^{3}$, Abed Hussain Khan ${ }^{4}$, Quazi Mamtaz Uddin Ahmed ${ }^{5}$

\begin{abstract}
We are reporting a case of cutaneous sarcoidosis with asymptomatic systemic involvement evident by investigation. Management of cutaneous sarcoidosis is challenging. However, maculopapular rash, which usually show better prognosis respond promptly by high dose of systemic steroid with gradual tapering dose.
\end{abstract}

Key words: Cutaneous sarcoidosis, maculopapular rash, bilateral hilar lymphadenopathy.

\section{Case Report}

A 45-year-old Bangladeshi man presented with skin rash and bilateral leg oedema with generalized lymphadenopathy including inguinal and both axillary regions. The largest

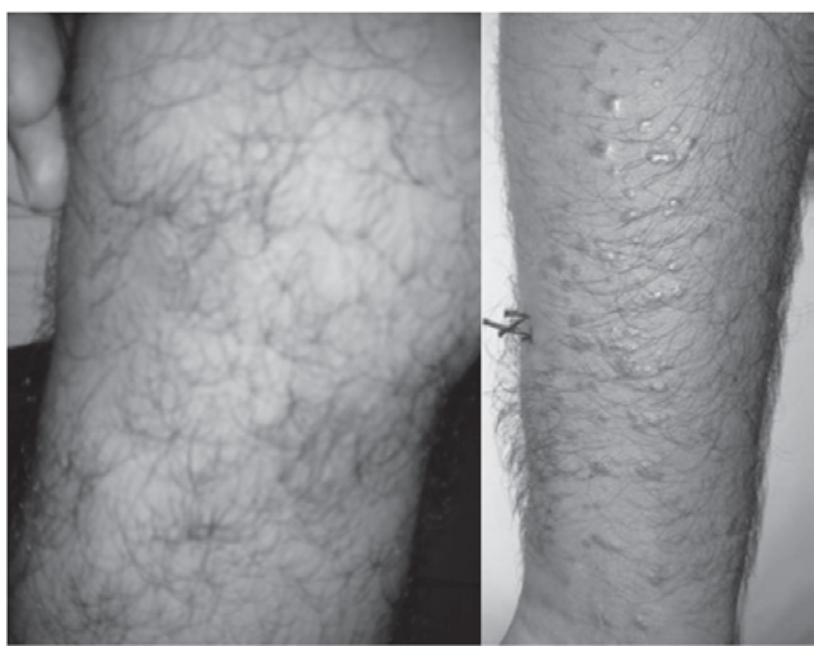

Fig.1: Maculopapular rash on both feet.

1. Dr. Mohammad Mainul Hasan Chowdhury, MBBS Post-graduate Student

2. Dr. Farzana Shumy, MBBS, FCPS (Medicine) Medical Officer

3. Dr. Probal Sutradhar, MBBS

Post-graduate Student

4. Dr. Abed Hussain Khan, MBBS, FCPS (Medicine) Medical Officer

5. Dr. Quazi Mamtaz Uddin Ahmed, MBBS, FCPS (Medicine) Associate Professor

*All the authors work in the Department of Internal Medicine, BSMMU, Shahbagh, Dhaka-1000, Bangladesh.

\section{Corresponding author:}

Dr. Mohammad Mainul Hasan Chowdhury, MBBS Post-graduate Student, Department of Internal Medicine, BSMMU, Shahbagh, Dhaka-1000, Bangladesh.

E-mail: shaown_imc@yahoo.com bilateral leg oedema for 3 months. On examination, he had lymph node was in the right inguinal region. There was presence of generalized, discrete, maculopapular rash with erythematous margin more markedly distributed in both upper and lower limbs [Fig.1]. Chest x-ray revealed bilateral hilar lymphadenopathy with bilateral pulmonary inflammatory lesion [Fig.2]. Complete blood count (CBC) revealed Haemoglobin-13 gm/dL, WBC- 4500/cmm (N-66\%, L-19\%), platelet count- $260000 / \mathrm{cmm}$, ESR- $60 \mathrm{~mm}$ in the $1^{\text {st }}$ hour, with raised C-reactive protein (CRP) $12.8 \mathrm{mg} / \mathrm{l}$. Peripheral blood film showed dimorphic anaemia with anisocytosis. Fasting blood sugar was $4.3 \mathrm{mmol} / \mathrm{L}$ and urine $\mathrm{R} / \mathrm{M} / \mathrm{E}$ revealed trace proteinuria but serum creatinine was $1.4 \mathrm{mg} / \mathrm{dl}$. USG of whole abdomen revealed- multiple enlarged para-aortic and peripancreatic lymph nodes, mild hepatomegaly with fatty change (grade-I). Liver function tests (LFT) were normal. Serum albumin was $33 \mathrm{gm} / \mathrm{L}$, lactate dehydrogenase (LDH)$266 \mathrm{U} / \mathrm{L}$ (normal up to 400), serum calcium- $9 \mathrm{mg} / \mathrm{dl}$ and Mantoux test was negative $(2 \mathrm{~mm})$. HRCT revealed nodular attenuated areas with septal thickening involving different segment of both lung. There was also presence of subcarinal lymph node and bilateral hilar lymphadenopathy. Lymph

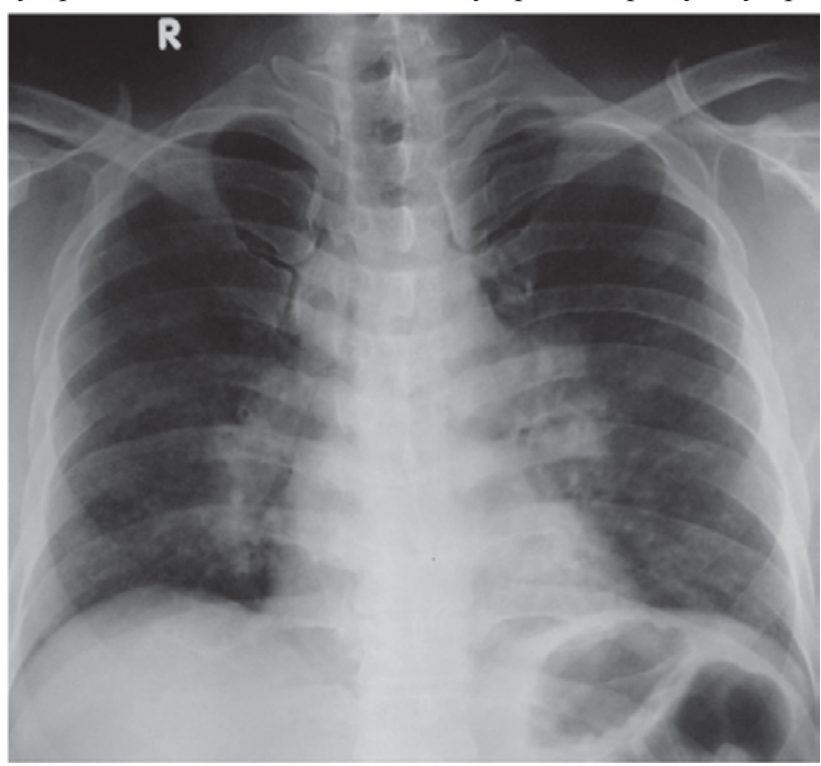

Fig.2: Chest $x$-ray showing bilateral hilar lymphadenopathy. 
node and skin biopsy showed many discrete non-caseating granuloma suggestive of sarcoidosis. Patient was discharged with local and systemic steroid $30 \mathrm{mg}$ daily and followed up four week later. He showed significant improvement and steroid was tapered.

\section{Discussion}

Sarcoidosis is a multi-system disease of unknown etiology that has a wide variety of clinical manifestations and frequently, an unpredictable course. It involves mainly the lungs, mediastinal and peripheral lymph nodes, skin, liver, spleen, eyes and parotid glands. Less frequently, but usually severe, manifestations also occur in the central nervous system, heart, upper respiratory tract and bones. Because lesions can exhibit much different morphology, cutaneous sarcoidosis is known as one of the great limitators in dermatology $y^{1-3}$. Cutaneous involvement in sarcoidosis may occur at any stage of the disease but most often present at the onset and the patients are often seen initially by a dermatologist. ${ }^{2,3}$ According to Mañá $\mathrm{J}$ et al, 70\% patients of sarcoidosis with cutaneous manifestation had other systemic manifestations at the time of presentation but almost one-third present with only skin lesions, without any other sign of sarcoidosis. They had also noticed that skin eruptions were accompanied by chest radiographic changes corresponding to stage I disease in $50 \%$ of the cases. In addition, some patients with systemic sarcoidosis may develop their skin manifestations later in the disease course. ${ }^{4}$ Veien et al reported no relationship between the extent of cutaneous manifestations and the extent of systemic disease in their study population consisting of 200 Caucasians patients diagnosed with cutaneous sarcoidosis. They found many of the infiltrative lesions appeared to be of a chronic nature and were commonly associated with pulmonary changes, such as mottling and fibrosis. ${ }^{5}$ In patients with skin lesions, other than Veien, Olive and Kataria found splenomegaly, hepatomegaly and lymphadenopathy in approximately one-third of the cases. ${ }^{6}$ The prevalence of a particular type of cutaneous lesion varies among races as well as individual cases. The frequency of specific cutaneous involvement in sarcoidosis ranges from $9 \%$ to $37 \% .^{5,7}$ Although different series report variable incidences of skin involvement during the course of the disease, nearly a quarter of sarcoidosis patients have occurrence of skin lesions. ${ }^{7}$ Skin lesions are divided into two groups, specific skin lesions where histological examination shows the typical sarcoid granulomas, and nonspecific skin lesions. Specific lesions are lupus pernio (LP), infiltrated plaques, maculopapular eruptions, subcutaneous nodules and scars, and rare morphologies such as alopecia, ulcers, hypopigmented patches, and ichthyosis. Despite the clinical importance of scar infiltrates in the diagnosis of sarcoidosis, it is often overlooked because the lesions are usually small and asymptomatic. Erythema nodosum is the most frequent nonspecific skin lesion in sarcoidosis.,7 Maculopapular lesions have been reported to be the most common cutaneous manifestation of granulomatous involvement in sarcoidosis. ${ }^{8}$ The papules are commonly red-brown to purple, slightly infiltrated and measure less than $10 \mathrm{~mm}$. In contrast to lupus pernio or plaques, they are more often associated with a favourable outcome and can resolve in rather a short time. They may even herald the disease and can be observed almost anywhere, often in groups. However, papules and nodules may also be seen in subacute and occasionally chronic phases of sarcoidosis. In general, skin manifestations are associated with less delay in diagnosis than other manifestations of sarcoidosis. ${ }^{9}$ The treatment of cutaneous sarcoidosis is often frustrating, because lesions may be refractory to treatment or may recur following successful treatment. For localized involvement, topical or intralesional steroids are used. Physicians frequently attempt to use superpotent topical steroids because of their occasional effectiveness. However, these steroids often do not adequately penetrate the skin lesion. Intralesional steroids (e.g., triamcinolone acetonide in a dosage of $5 \mathrm{mg} / \mathrm{mL})^{10}$ are typically more effective, with injections repeated at two- to three-week intervals. Systemic agents are reserved for widespread, progressive lesions or those that impair function. Systemic glucocorticoids are the most effective agents. ${ }^{11}$ They are commonly used at slow, tapering dosages, starting at 20 to $40 \mathrm{mg}$ of oral prednisone daily for four to six weeks. ${ }^{12}$ However, there are many drawbacks in this therapy. Aside from the well-known complications of chronic steroid use, not all patients respond to systemic steroids. Those who do respond frequently experience flare-ups of disease when the treatment is completed. Many other medications may be used in refractory cases, including such agents as hydroxychloroquine ${ }^{13}$ and methotrexate ${ }^{14}$. Even these drugs can be used as monotherapy in steroid resistant cases or in patient unable to tolerate steroids. Despite universal acceptance these treatments can be failed. In such situations, innovative treatment options can be considered like tumour necrosis factor- alpha inhibitors infliximab and adalimumab. Although early reports are promising, experience is limited. In some reported cases Isotretinoin has been used successfully. Melatonin and allopurinol are not well studied in cutaneous sarcoidosis. Thalidomide has limited, albeit promising, supporting data. Ciclosporin and chlorambucil have been largely abandoned because of their toxicity. Laser therapy became newer promising modality. ${ }^{15}$

\section{References:}

1. Kenneth E, Kataria YP. Cutaneosus Manifestations of Sarcoidosis. Arch Intern Med 1985; 145:1811-1814.

2. Lodha S, Sanchez M, Prystowsky S. Sarcoidosis of the Skin. Chest 2009; 136:583-586.

3. Sharma OP. Sarcoidosis: a Historical Perspective. Clin Dermatol 2007; 25(3):232-241.

4. Mañá J, Marcoval J, Graells J, Salazar A, Peyri J, Pujol R. Cutaneous Involvement in Sarcoidosis. Relationship to Systemic Disease. Arch Dermatol 1997;133: 882-888.

5. Veien NK, Stahl D, Brodthagen H. Cutaneous Sarcoidosis in Caucausians. J Acad Dermatol 1987;16: 534-540.

6. Olive KE, Kataria YP. Cutaneous Manifestations of Sarcoidosis: Relationship to Other Organ System Involvement, Abnormal Laboratory Measurements, and Disease Course. Arch Intern Med 1985;145:1811-1814.

7. Rose AS, Tielker MA, Knox KS. Hepatic, Ocular and Cutaneous Sarcoidosis. Clin Chest Med 2008;29(3):509-523. 
8. English JC 3rd, Purvisha JP, Patel PJ, Greer KE. Sarcoidosis. J Am Acad Dermatol 2001;44 725-743.

9. Judson MA, Thompson BW, Rabin DL, et al. The Diagnostic Pathway to Sarcoidosis. Chest 2003;123:406-412.

10. Khatri KA, Chotzen VA, Burrall BA. Lupus Pernio: Successful Treatment with a Potent Topical Corticosteroid. Arch Dermatol 1995;131:617-618.

11. Samtsov AV. Cutaneous Sarcoidosis. Int $J$ Dermatol 1992;31:385-391.

12. Sharma OP. Sarcoidosis of the Skin. In: Freedberg IM, Fitzpatrick TB, eds. Fitzpatrick's Dermatology in General Medicine. 5th edn. New York: McGraw-Hill, 1999:2099-2106.
13. Zic JA, Horowitz DH, Arzubiaga C, King LE. Treatment of Cutaneous Sarcoidosis with Chloroquine. Review of the Literature. Arch Dermatol 1991;127:1034-1040.

14. Webster GF, Razsi LK, Sanchez M, Shupack JL. Weekly Low-Dose Methotrexate Therapy for Cutaneous Sarcoidosis. J Am Acad Dermatol 1991;24:451-4.

15. Doherty CB, Rosen T. Evidence-Based Therapy for Cutaneous Sarcoidosis. Drugs 2008;68(10):1361-83. 